\title{
A Review and Conjectures of Therapies for Depression
}

\author{
Jiang Aiyang*1 \\ ${ }^{1}$ Chengdu Experimental Foreign Languages School, China \\ *Guanghua.ren@gecacademy.cn
}

\begin{abstract}
In this review, various treatment methods of depression, corresponding to their different symptoms and conditions have been reviewed. Also, study for the inflammatory mechanism, through stem cell therapy, MECT treatment (severe ones), and conventional drug treatment, analysis for the targets and variation sites of different kinds of inflammation, inhibit the development of the disease have been presented. In the future, the development of treatment methods will transfer to gene therapy.
\end{abstract}

Keywords: "Depression", "Gene-editing technology", "Artificial intelligence”, "Data analysis"

\section{INTRODUCTION}

In recent years, the incidence of depression is kept increasing among teenagers, especially in China. But sometimes the cause of the depression is not only gene but also the surrounding environment. Scientists have already figure out some therapies for curing the depression, but there is still a plethora of patients who suffer from this kind of disease. By learning the mechanism and different symptoms of this disorder, there are also some conjectures of new treatment. The effectiveness and risk need further study.

\section{SYMPTOMS AND MECHANISM OF DEPRESSION}

This part describes the basic symptoms and mechanism of depression that were previously discovered by scientists and doctors.

\subsection{Symptoms}

The symptoms of depression are separated into three different parts: Core symptom group, Psychological symptom group, and Physical symptom group. First, for the core symptom group patients will always feel upset and don't change with the surrounding environment. Also, they will lose interest and passion in everything. They do some simple things just for killing time but not for feeling happy or to find their interest. Second, for psychological symptom group, patients will always feel anxious. They will say less and think slowly.[1] Their memory will start to decline and their attention is started to be unusual. They will think that they are useless to society, they are always alone and no one desires to help them, and they will feel hopeless about everything especially for their future. They will magnify their mistakes and think they are good at nothing. More severe patients will have suicidal tendencies and the action of suicide. These severe patients sometimes don't want to get any help from others and reject any treatments. Third, for physical symptom groups, they always have insomnia and be tired all day long. And anorexia is a common symptom as well.[2]

\subsection{Mechanisms}

Depression has a complex mechanism and genetic basis. Nowadays the actual genetic basis of depression is still uncertain. But it will indeed be hereditary by genes. A report from Nature in 2014, according to Jonathan Flint and his colleagues, shows that with the comparison of 5303 patients' gene sequence and 5337 control group's gene sequence, they found out there is two mutation part because of depression and both of them are on the tenth chromosome. One of the mutation parts surrounds SIRT1 and another one is an intron of gene LHPP.[3] This shows that depression is not only a psychological disease but also a disease relating to the endocrine. These mutations lead the neurotransmitters not to be released properly.

\section{NORMAL THERAPIES}

This part talks about some therapies for depression that have already been used in clinical treatment. 


\subsection{Drugs}

Depression cannot be fully cured. Patients can only get treatment to alleviate symptoms and sometimes they will think they are cured but they are very likely to relapse. The common clinically used drugs for doctors are SSRIs, SNRIs, and NDRIs. For SSRIs and SNRIs, they selectively re-uptake 5-hydroxytryptamine and have less impact on Noradrenaline and Dopamine.[4] But NDRIs are different. They help to re-uptake Noradrenaline and Dopamine. Although the impact will be small, they will still help the patients a lot. All of these drugs have their adverse reaction and patients will have a dependence. So with those adverse reactions some of the patients feel even worse and for teenagers who take SSPIs, they will be more likely to have suicidal tendencies and actions.[5] In the worst situation, after the treatment of depression, some of the patients will turn to Bipolar Disorder or switch to major depression. Drug intervention is not the only treatment, patients should also be relaxed and can't be stimulated. The surrounding environment can help them to reacquire their happiness and interest in the world.[6-8]

\subsection{Nervous stem cells}

Is to use nervous stem cells and induced pluripotent stem cell to model the nervous system of human to better recognize the mechanism of depression. The technology of iPSC can imitate the effect of drugs. But it can treat depression in a more careful way. By figuring out its mechanism, iPSC produces new cells to replace the old ones and cure the disease.[9]

\subsection{MECT for severe cases}

MECT is also a way of curing major depression. Patients suffering from severe depression, strong selfharm, suicidal behavior, and obvious self-blame and self-guilt are particularly suitable. It also reminds us that patients with severe depression should choose electroconvulsive therapy as the current international recognition. This therapy has a huge side effect. Patients may feel like losing their emotions and it will cause memory loss. Some of the patients who take the treatment will relapse not too long after the treatment.[10]

\section{CONJECTURES OF GENE EDITING THERAPY}

With the development of gene-editing technology, there are more possibilities for depression to be cured.

Normally, to treat depression doctors always give the same drugs at the beginning to patients. After a period of observation if the drugs work the doctor will keep using these kinds of drugs. If they don' $t$ work the doctor will change different drugs and observe again until finding the most effective drugs for patients. This process will take a long time and always have many drawbacks. Some patients just abandon treatment. And some situations are even worse. Many patients can' t endure the side effect of the drugs and have suicidal thoughts. If patients can do the gene test and find out the mutation on the nucleotide. Doctors can edit the mutated site. They will be cured and will not affect their next generation. The mechanism of a genetic-based disease is that the mutation on the mRNA causes the change of its correspondence on the nucleobase and change the DNA. The change in the DNA will then change the expression of proteins. And finally, we can see the symptoms. The special mechanism of depression is called BDNF Brainderived neurotrophic factor. Doctors can do the alignments of mRNA and the protein to find out that particular mutated site and design the guide RNA in CRISPR to correct it.[11] 
1. CRISPR
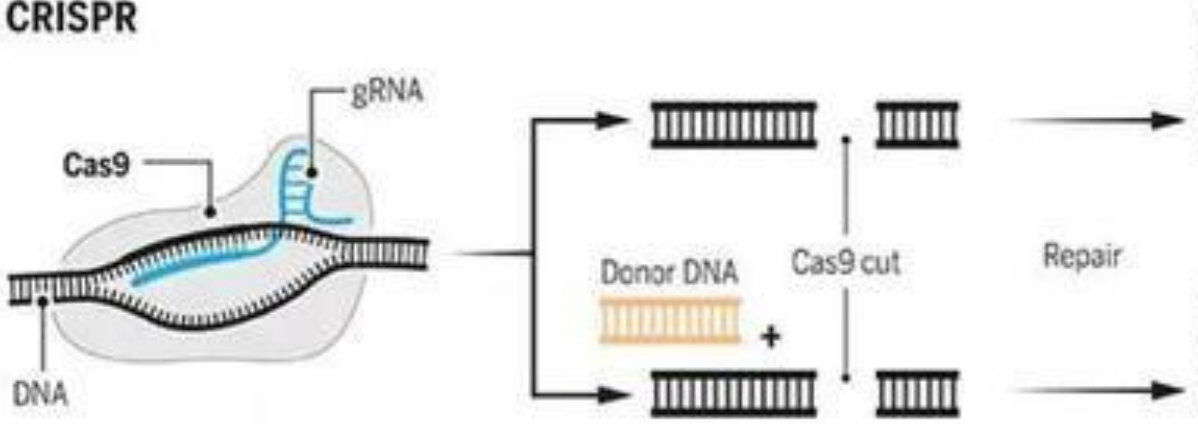

Non-homologous end joining

IIIIIIIIIIIII $\cdot$ IIIIII Insertions/deletions

\section{DNA base editing}
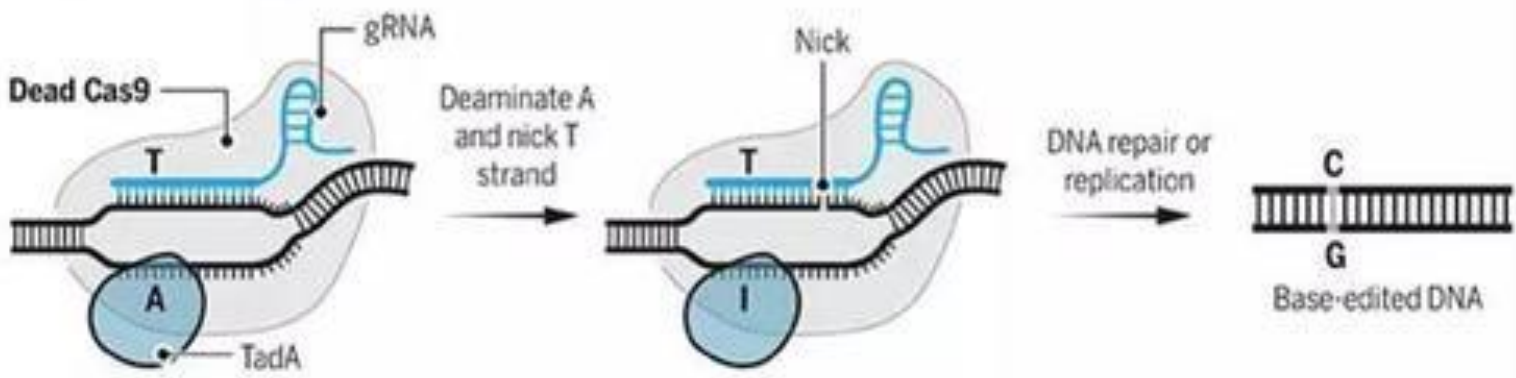

\section{RNA base editing}

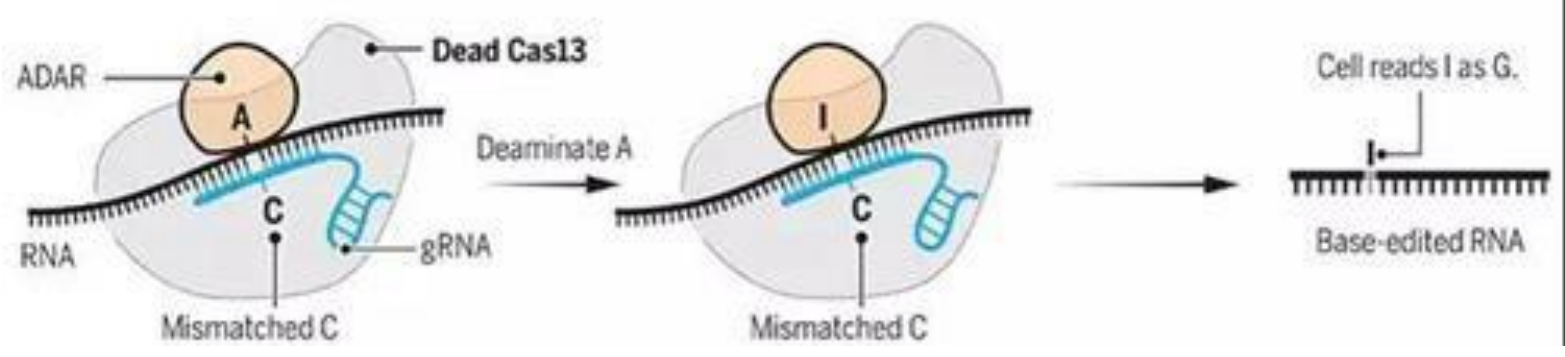

Figure 1 Comparison of CRISPR, DNA base editing and RNA base editing techniques[12-13]

Everyone knows about AI artificial intelligence and competitions between Alphago and professional go players.[14] When people are astonished by $\mathrm{Al}$ ' $\mathrm{s}$ capability of learning I' $m$ thinking that if we can use AI to do the test, we' 11 get a more accurate result by its calculation. AI is just like the human brain it learns things from different examples. This means that if we want to use AI to increase the rate of success we still need a large database that must be based on numerous experiments. Then there' $\mathrm{s}$ this ethical problem again, as who will desire to be the object of the experiment, especially for these high risky gene-related experiments.

There' $s$ one machine called BioXP which was designed by Dan Gibson and his team 7 years ago.[15] In 2013 when China was in the pandemic of H7N9, WHO asked his group to help China by printing the DNA of the virus to develop the vaccine. By downloading and printing the DNA sequence which was published by China government on the internet, his group used less than 12 hours to produce the DNA of the virus and their collaborators at Novartis turned the synthetic DNA into a flu vaccine. This whole process took less than one day. Synthetic DNA is also a kind of gene-editing technology. Its essence is to add different pieces of nucleobase together and create the DNA. Using these synthetic cells to figure out the side effect of gene-editing might be the choice. With the DNA sequence, we can produce whatever cells we want. So that if we can produce all body cells and germ cells in the human body, we can do several experiments and observations. Then there will be a large database that can be learned by AI. As the result, we can figure out almost every situation we will meet when doing the gene-editing operation. Some people may worry about the risk they will take when editing the gene. However, every operation has a relative risk, as the technology is matured, the risk of gene-editing will be the same as other normal operations. Once this technology is implemented, its possibility is endless.

Nowadays, there are a lot of students suffering from depression. Some of them have the suicidal thinking, and depression causes a lot of death in school. It is hard 
for school to deal with these cases and handle such cases properly with family members. With the delayed testing and diagnosis, some of the family members do not believe that their children have the psychological diseases, and most of them think that the school causes the death. In the future, everyone should get the regular psychological check-ups. Patients must follow the doctors' advice to get proper therapies.

\section{CONCLUSION}

Depression is not fully understood by us. Or all of the mental illnesses are not fully understood. There is still a long way to go and a lot to research. Gene editing technology will be widely used in the future as scientists figure out its risks and particularity. As the ethical problem is handled, it will be more developed and cutting-edge. Genetic modification will become the trend of future medical treatment. Based on the technology of BioXP and artificial intelligence which rely on big data analysis, gene editing technology will be grown at a much quicker speed and with a better future prospect. In the future, with the development of medical technology, normal therapies will have a higher rate for curing the depression and less side-effect. It is hard to say that gene-editing technology will be used in the future clinic treatment. But scientists should still working on this kind of technology due to its possible effectiveness.

\section{REFERENCES}

[1] Allegra S. Anderson, Kelly H. Watson,Michelle M. Reising, Jennifer P. Dunbar,Alexandra H. Bettis, Meredith A. Gruhn, Bruce E. Compas. Relations Between Maternal Coping Socialization, Adolescents' Coping, and Symptoms of Anxiety and Depression[J]. Journal of Child and Family Studies,2021(prepublish).

[2] Lama Majed Al Qaisy, Rihan T Tarawneh. Alleviate the Symptoms of Depression among Clients in Mental Health Rehabilitation Clinic at Royal Medical Services[J]. World Journal of Social Science,2021,8(1).

[3] Zhiyang Liu, Wenjun Su, Wenjie Yan, Jing Hu, Ping Chen, Zhiyong Cao. Journal of Clinical Medicine,2020,48(12):1513-1516. (in Chinese with English abstract)

[4] The ABCs of antidepressants. SSRIs, SNRIs, NDRIs: what's the difference?[J]. Health after 50 with Scientific American Consumer Health,2016,27(15).

[5] Kautzky Alexander, James Gregory M, Philippe Cecile, Baldinger-Melich Pia, Kraus Christoph, Kranz Georg S, Vanicek Thomas, Gryglewski
Gregor, Hartmann Annette M, Hahn Andreas, Wadsak Wolfgang, Mitterhauser Markus, Rujescu Dan, Kasper Siegfried, Lanzenberger Rupert. Correction: Epistasis of HTR1A and BDNF risk genes alters cortical 5-HT1A receptor binding: PET results link genotype to molecular phenotype in depression.[J]. Translational psychiatry,2019,9(1).

[6] Horstmann Sonja, Binder Elisabeth B Pharmacogenomics of antidepressant drugs.[J]. Pharmacology \& therapeutics,2009,124(1).

[7] A. Marais, E. Osuch. The Pharmacological management of depression[J]. SA Pharmaceutical Journal,2019,86(2).

[8] Uçan Gündüz Gamze, Parmak Yener Neslihan, Kılınçel Oğuzhan, Gündüz Cem. How Does Usage of Serotonin Noradrenaline Reuptake Inhibitors Affect Intraocular Pressure in Depression Patients?[J]. Journal of ocular pharmacology and therapeutics : the official journal of the Association for Ocular Pharmacology and Therapeutics,2018,34(4).

[9] Jiang Ruru, Li Xin, Ha Xiaoqin. The role of stem cells in the treatment of depression [J]. Chinese Journal of Bio-products,2020,33(12):1450$1453+1459$.

[10] Pang Y. Effect of MECT combined with small dose of olanzapine on patients with depression and suicidal ideation [J]. Journal of Medical Theory and Practice, 2019,32(21):3451-3452.

[11] Perez-Pinera, P., et al. (2013). "RNA-guided gene activation by CRISPR-Cas9-based transcription factors." Nat Methods 10(10): 973-976.

[12] C. BICKEL/SCIENCE; (DATA) D. B. T. COX ET AL., SCIENCE 358, 6362, 2017 (C) SCIENCE; J. DOUDNA AND E. CHARPENTIER, SCIENCE 346, 6213, 2014

[13] SCIENCE; GAUDELLI ET AL., NATURE 551, 7677,2017

[14] Martínez Plumed Fernando, Gómez Emilia, Hernández Orallo José. Futures of artificial intelligence through technology readiness levels[J]. Telematics and Informatics,2021,58.

[15] Claudia H Alvarez. DNA assembly and cloning in an overnight run with the BioXp ${ }^{\mathrm{TM}} 3200$ system[J]. Nature Methods: Techniques for life scientists and chemists,2015,12(12). 